\title{
Jugular foramen tumors: diagnosis and treatment
}

\author{
Ricardo Ramina, M.D., Joao Jarney Maniglia, M.D., \\ Yvens Barbosa Fernandes, M.D., Jorge Rizzato Paschoal, M.D., \\ Leopoldo Nizan Pfeilsticker, M.D., Maurício Coelho Neto, M.D., \\ AND GUILHERME BORGES, M.D.
}

\author{
Neurosurgery Department of Instituto de Neurologia de Curitiba; and Departments of Neurosurgery \\ and Ear, Nose, and Throat, State University of Campinas, Brazil
}

\begin{abstract}
Object. Jugular foramen tumors are rare skull base lesions that present diagnostic and complex management problems. The purpose of this study was to evaluate a series of patients with jugular foramen tumors who were surgically treated in the past 16 years, and to analyze the surgical technique, complications, and outcomes.

Methods. The authors retrospectively studied 102 patients with jugular foramen tumors treated between January 1987 and May 2004. All patients underwent surgery with a multidisciplinary method combining neurosurgical and ear, nose, and throat techniques. Preoperative embolization was performed for paragangliomas and other highly vascularized lesions. To avoid postoperative cerebrospinal fluid (CSF) leakage and to improve cosmetic results, the surgical defect was reconstructed with specially developed vascularized flaps (temporalis fascia, cervical fascia, sternocleidomastoid muscle, and temporalis muscle). A saphenous graft bypass was used in two patients with tumor infiltrating the internal carotid artery (ICA). Facial nerve reconstruction was performed with grafts of the great auricular nerve or with 12th/seventh cranial nerve anastomosis. Residual malignant and invasive tumors were irradiated after partial removal.

The most common tumor was paraganglioma (58 cases), followed by schwannomas (17 cases) and meningiomas (10 cases). Complete excision was possible in 45 patients $(77.5 \%)$ with paragangliomas and in all patients with schwannomas. The most frequent and also the most dangerous surgical complication was lower cranial nerve deficit. This deficit occurred in 10 patients (10\%), but it was transient in four cases. Postoperative facial and cochlear nerve paralysis occurred in eight patients (8\%); spontaneous recovery occurred in three of them. In the remaining five patients the facial nerve was reconstructed using great auricular nerve grafts (three cases), sural nerve graft (one case), and hypoglossal/facial nerve anastomosis (one case). Four patients (4\%) experienced postoperative CSF leakage, and four $(4.2 \%)$ died after surgery. Two of them died of aspiration pneumonia complicated with septicemia. Of the remaining two, one died of pulmonary embolism and the other of cerebral hypoxia caused by a large cervical hematoma that led to tracheal deviation.

Conclusions. Paragangliomas are the most common tumors of the jugular foramen region. Surgical management of jugular foramen tumors is complex and difficult. Radical removal of benign jugular foramen tumors is the treatment of choice, may be curative, and is achieved with low mortality and morbidity rates. Larger lesions can be radically excised in one surgical procedure by using a multidisciplinary approach. Reconstruction of the skull base with vascularized myofascial flaps reduces postoperative CSF leaks. Postoperative lower cranial nerves deficits are the most dangerous complication.
\end{abstract}

\section{KEY WORDS - jugular foramen • paraganglioma • schwannoma • meningioma • cranial base reconstruction - cerebrospinal fluid leak}

Jugular foramen tumors are deeply located, may be highly vascularized, and involve important neurovascular structures and bone at the cranial base. Management of these lesions requires understanding of diagnosis, anatomy, natural history, postoperative complications, and cooperation among multiple disciplines. Advances in skull base surgery, interventional radiological procedures, postoperative care, and rehabilitation have improved the treatment of these tumors significantly.

Paragangliomas are the most common jugular foramen

Abbreviations used in this paper: $\mathrm{CA}=$ carotid artery; $\mathrm{CSF}=$ cerebrospinal fluid; $\mathrm{CT}=$ computerized tomography; ICA = internal CA; IJV = internal jugular vein; $\mathrm{MR}=$ magnetic resonance; $\mathrm{SCM}=$ sternocleidomastoid. tumor. These lesion have been called chemodectomas, nonchromaffin tumors, and glomus tumors. These neoplasms are highly vascularized and histologically benign in most cases, but may invade bone, blood vessels, dura mater, and cranial nerves. Pulsatile tinnitus and hearing loss are the most frequent presenting symptoms. Schwannomas and meningiomas are the next most frequent jugular foramen neoplasms. Invasive tumors may present with lower cranial nerve deficits as the initial symptom. Pain in the ear and temporal region associated with paralysis of cranial nerves are symptoms of malignant lesions.

A series of 102 patients with jugular foramen tumors that were surgically treated between January 1987 and May 2004 is presented. All tumors originated in the jugular foramen region and were surgically treated using the 
same multidisciplinary otoneurosurgical approach. The objective of treatment was total removal with preservation of cranial nerves and vessels. Tumor size, biological behavior, histological features, invasion of cranial nerves and bone of the cranial base, and extension into the cavernous sinus, clivus, and brainstem were factors limiting radical resection. Surgical complications and outcomes are presented.

\section{CLINICAL MATERIAL AND METHODS Patient Population}

One hundred two patients with jugular foramen tumors were surgically treated between January 1987 and May 2004 at the Neurological Institute of Curitiba and the State University of Campinas in Brazil. The most frequently seen jugular foramen tumor in this series was paraganglioma (58 cases [56.8\%]), followed by lower cranial nerve schwannomas (17 cases [16.6\%]) and meningiomas (10 cases [9.8\%]). There were 77 women $(76 \%)$ and 25 men (24\%). In the group with paragangliomas there were 47 women and 11 men (approximately a 4:1 female/male ratio) whose mean age was $42.5 \pm 12$ years (range 18-72 years).

The most common initial clinical symptoms of paragangliomas were pulsatile tinnitus and hearing loss. Two patients presented with bilateral jugular foramen paragangliomas and two presented with carotid body tumors. Two tumors secreted catecholamines (norepinephrine). Secreting tumors require careful perioperative treatment with $\beta$ blockers to avoid complications of catecholamine overload. Three patients related a family history of jugular foramen or carotid body tumors, and two of these patients presented with bilateral jugular foramen paragangliomas. Four tumors were malignant, with metastases to the cervical lymph nodes. The aggressive and malignant tumors as well as the meningiomas presented initially with lower cranial nerve palsy. In the meningioma group, two tumors were histologically malignant, and four presented with malignant behavior. Most patients with malignant tumors reported pain in the ear and mastoid region. Eleven patients $(10.8 \%)$ had undergone surgery previously at other institutions.

Preoperative high-definition CT scans were obtained for analysis of bone structures of the cranial base, tumor calcification, hyperostosis, and bone erosion. Preoperative MR imaging with addition of Gd as well as MR angiography was performed to study tumor vascularization, extension, and its relationship to neighboring structures. Venous circulation and occlusion of the sigmoid sinus were studied with MR angiography. Digital subtraction angiography was performed for diagnosis and to guide preoperative embolization in cases of highly vascularized lesions.

Embolization was performed 3 to 5 days before the surgical procedure. Tumor feeding vessels (ascending pharyngeal, internal maxillary, and occipital arteries, carotid tympanic branches, and the posterior inferior cerebellar artery) were embolized with Gelfoam and/or Ivalon particles. Balloon test occlusion was performed when the tumor involved or invaded the ICA. High-flow saphenous bypass grafts were placed to reconstruct the ICA in two patients before tumor removal and sacrifice of this vessel. The same surgical procedure was used to treat all patients.
Postoperatively the patients underwent follow-up with MR imaging evaluation every 6 months.

\section{Surgical Procedure}

Position of Patient and Skin Incision. After induction of general anesthesia, the patient is placed in the dorsal position with the head held in a Mayfield clamp and turned $45^{\circ}$ to the opposite side. The opposite jugular vein must be free from compression. A nasogastric tube is inserted and intraoperative monitoring of facial and lower cranial nerves is performed. All contact areas are protected with foam pads or water bags. The skin incision is C-shaped, starting in the temporal region and circumscribing the ear as far as the anterior border of the SCM muscle (Fig. 1). The great auricular nerve is identified, and in most cases is cut just behind its bifurcation over the parotid gland. This nerve may be preserved in small tumors and if necessary is used as a graft to reconstruct the facial nerve.

The external auditory canal and the ossicular chain are preserved when the tumor has not invaded or destroyed these structures. If the patient is already deaf, the external auditory canal is cut at the osteocartilaginous junction. The incision is closed in two layers and the posterior auricular muscle is dissected and folded over the suture line to obtain a watertight closure of the external auditory canal.

Reconstruction of the Cranial Base. To avoid postoperative CSF leaks and obtain good cosmetic results, a technique for reconstruction of the cranial base with closure of the wound in three layers was developed by our group. The dura mater (first layer) is closed, if possible, in a watertight fashion. If the dura is infiltrated and must be resected, a graft of the temporalis fascia and fibrin glue are used to close the defect. The posterior half of the temporalis muscle is incised, dissected, turned down, and sutured in the cervical and parotid fasciae to cover the dura mater (second layer). This vascularized muscle flap fills the surgical defect in the mastoid cavity (Fig. 2). This strategy provides very good cosmetic results without use

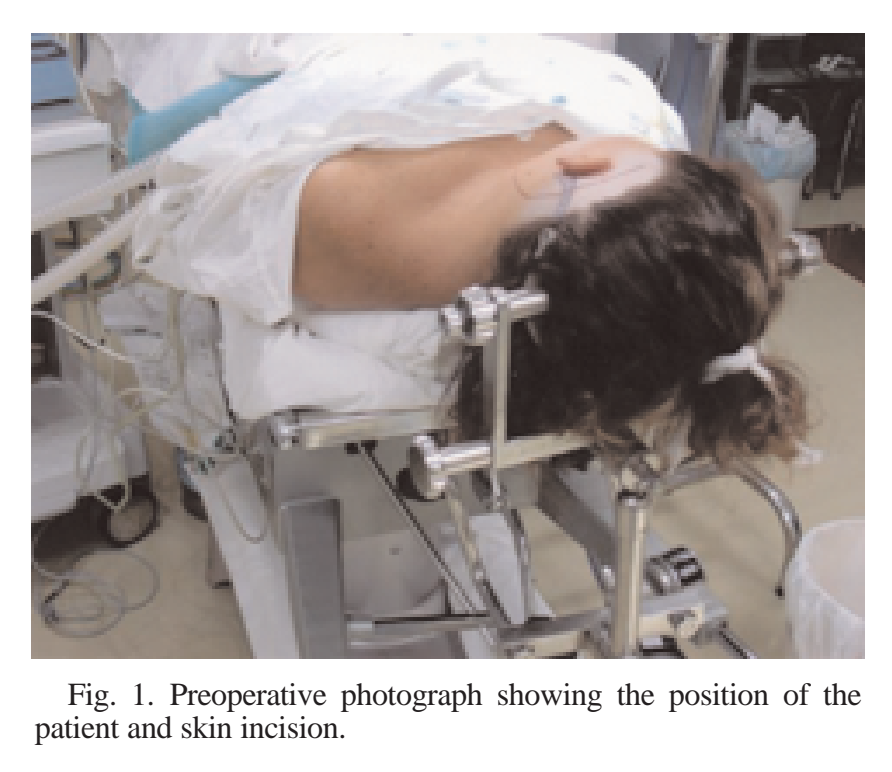

Neurosurg. Focus / Volume 17 / August, 2004 


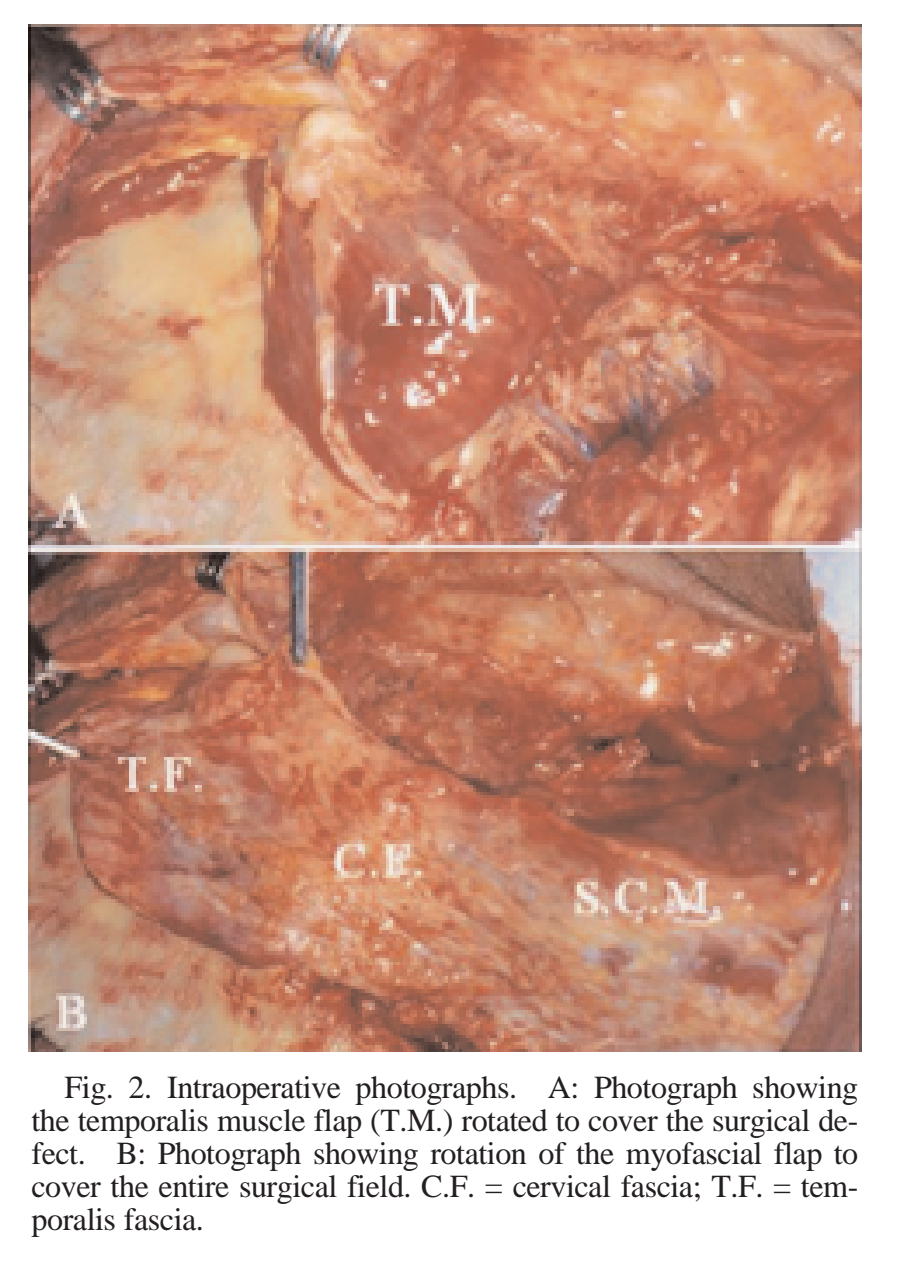

of abdominal fat graft. The temporalis fascia, cervical fascia, and SCM are used as the third vascularized layer. The temporalis muscle fascia is incised and dissected inferiorly as far as the temporal line. The cervical fascia is cut posterior to the external auditory canal, mastoid tip, and over the SCM. The insertion of the SCM is removed from the mastoid. This flap is secured with sutures in the temporalis fascia and in the parotid and cervical fascia to cover the temporalis muscle flap and the entire surgical field (Fig. 2).

Neck Dissection. The anterior border of the SCM is dissected and the major vessels of the neck (common CA, ICA, external CA and its branches, and IJV) are identified. The digastric muscle is used as a guide for dissection of the 12th and seventh cranial nerves. The accessory nerve runs laterally to the IJV in the majority of cases and crosses the external CA inferiorly to the digastric muscle. The facial nerve exits the stylomastoid foramen superiorly to the digastric muscle. The vagus nerve and the sympathetic trunk running lateroinferiorly to the common CA are dissected. The vertebral artery is identified at the skull base after dissection of the lateral process of C-1 and the superior and inferior oblique muscles. The IJV is dissected and the common facial vein is closed with a suture/ligature and cut. This vessel is followed up to the jugular foramen and in cases of paragangliomas and some meningiomas may be filled with tumor.
Facial Nerve Management. The facial nerve is identified at the stylomastoid/parotid region. The mastoid tip, the posterior belly of the digastric muscle, the "pointer," and the tympanomastoid suture are the references for dissection of facial nerve (Fig. 3). This nerve is not removed from its bone canal when it is not infiltrated by the lesion. According to our experience this strategy will avoid postoperative facial paralysis by rerouting the seventh cranial nerve. Tumor removal is accomplished anteriorly and posteriorly to the facial canal. When the nerve is infiltrated, it is resected and reconstructed with grafts acquired from the great auricular or sural nerves.

\section{Temporal Bone Dissection}

Radical mastoidectomy is the next step (Fig. 4). The facial nerve canal, the labyrinth, mastoid antrum, ossicles, and the sinodural angle are dissected. The sigmoid sinus is exposed from the superior petrosal sinus to the jugular bulb and the retrofacial mastoid cells are removed. The posterior and anterior walls of the external auditory meatus are drilled to better expose the tumor in the ear. After removal of the tympanic membrane, the tumor within the ear, eustachian tube, and mastoid cells is removed. The tympanic bone is drilled to expose and control with bipolar coagulation the carotid-tympanic branches (ICA branches) that are difficult to embolize preoperatively.

Craniectomy and Opening of the Jugular Foramen. A retrosigmoid craniectomy $(3 \mathrm{~cm}$ in diameter) exposing the posterior fossa dura and the posterior portion of the jugular foramen is the next surgical step. After removal of the lateral process of $\mathrm{C}-1$, and removal of the posterior border of the jugular foramen with a drill and a small Kerrison punch, the jugular foramen is widely opened, exposing the tumor in the cervical region and jugular bulb (Fig. 5).

Removal of Extradural Tumor. The sigmoid sinus is ligated with two sutures inferiorly to the superior petrosal sinus (Fig. 6). The IJV is double-ligated (suture/ligature) and cut. The posterior wall of the sigmoid sinus is incised and the intraluminal portion of the lesion is dissected from the anterior wall of the sigmoid sinus and jugular bulb (Fig. 7). The IJV is opened and tumor dissection is performed beginning at the anterior jugular bulb wall. Pres-

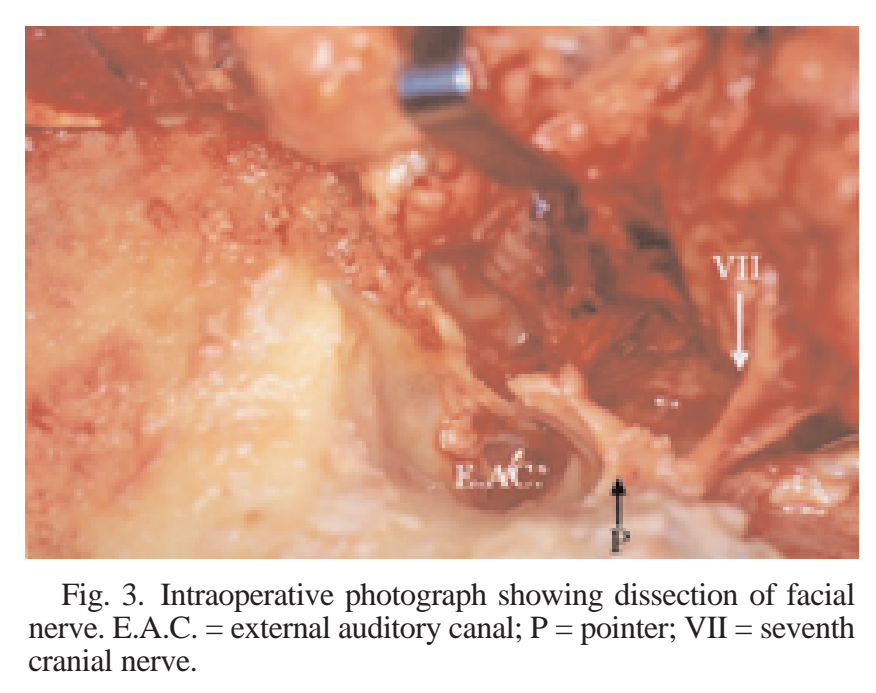




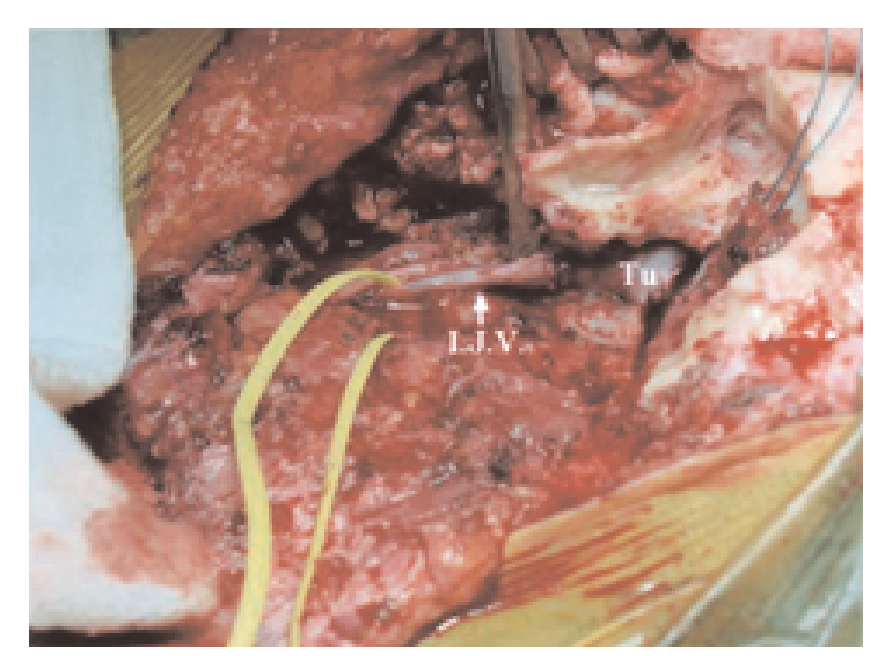

Fig. 4. Intraoperative photograph showing radical mastoidectomy. Tu. $=$ tumor in the jugular foramen.

ervation of the anterior jugular bulb wall is helpful in preserving the lower cranial nerves. After tumor removal, bleeding from the inferior petrosal sinus is controlled with Gelfoam or Surgicel. Anterior tumor extension displacing

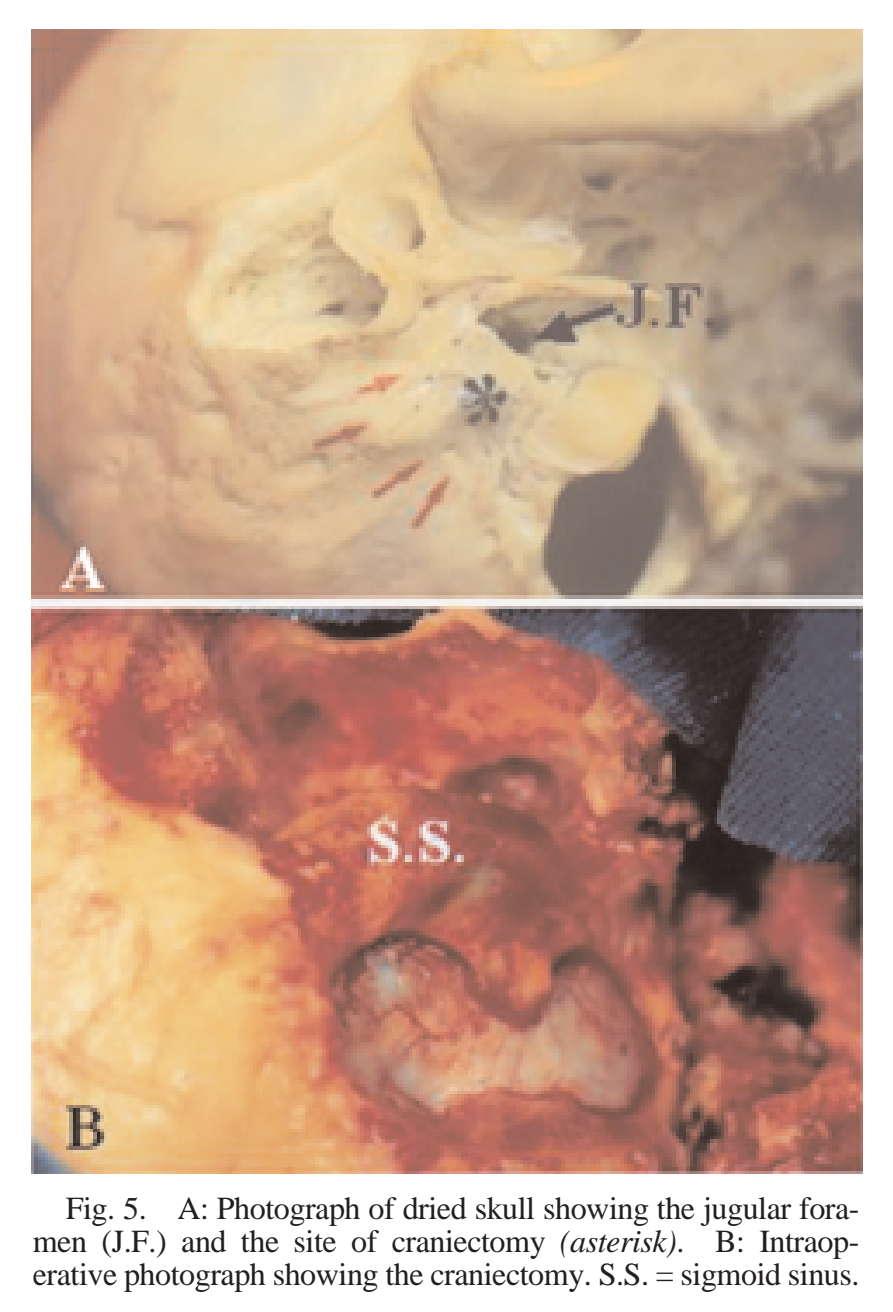

the ICA and tumor within the jugular bulb are removed with the aid of an operating microscope.

Intradural Tumor Removal. The medial wall of the sigmoid sinus is incised and the cerebellomedullaris cistern is opened. The intradural jugular foramen region is exposed with minimal cerebellar retraction. In small tumors the lower cranial nerves are easily identified at the brainstem. In these cases, if the nerves are not infiltrated, total tumor removal with preservation of these structures is accomplished. When the intradural portion of the lesion is large, compressing the brainstem, and the caudal cranial nerves cannot be identified, shrinkage of the tumor with bipolar coagulation is first performed and piecemeal intracapsular tumor resection helps to dissect the lower cranial nerves (Fig. 8).

Fascicles of the ninth, 10th, and 11th cranial nerves may be infiltrated and must be resected to achieve total removal. This should be done only if the patient presents preoperatively with paralysis of these nerves. Intraoperative monitoring of caudal cranial nerves is useful during dissection of this part of the tumor. Radical tumor removal that encompasses infiltrated bone and dura mater is attempted in all cases of benign noninvasive lesions. Malignant or aggressive tumors show no clear cleavage planes; total removal is not possible in these cases. The surgical defect is reconstructed with the technique described earlier. With this procedure no abdominal fat graft is used. Lumbar drainage is used only in cases in which the dural defect could not be closed in a watertight fashion.

\section{Postoperative Care}

In the intensive care unit the function of the lower cranial nerves should be carefully examined. Extubation is performed only after the patient is completely awake, to avoid aspiration due to dysfunction of caudal cranial nerves. The nasogastric tube is left in place until swallowing function can be assured. Tracheostomy should be performed when the patient presents with swallowing difficulty and recurrent aspiration.

\section{RESULTS}

\section{Postoperative Complications}

Paragangliomas (58 cases) were the most common tumors in this series, followed by schwannomas (17 cases), meningiomas (10 cases), chordomas and chondrosarcomas (six cases), malignant tumors (four cases), aneurysmal bone cysts (two cases), cholesteatomas (two cases), and chondroma, lymphangioma, and inflammatory granuloma (one case each). The tumor was totally removed in 74 cases $(73 \%)$ and in $86 \%$ of benign tumors. Radical removal of paragangliomas was possible in 45 patients $(77.5 \%)$. Three of these patients with radical resection presented with recurrences and underwent repeated surgery. In 13 paragangliomas (22.4\%) total resection was not possible due to invasion of lower cranial nerves and/or brainstem. Four of these tumors were malignant and three had previously been treated surgically elsewhere. Radiotherapy was performed for malignant and invasive tumors. Radical removal was possible in all cases of schwannomas and in five patients with benign meningiomas. 


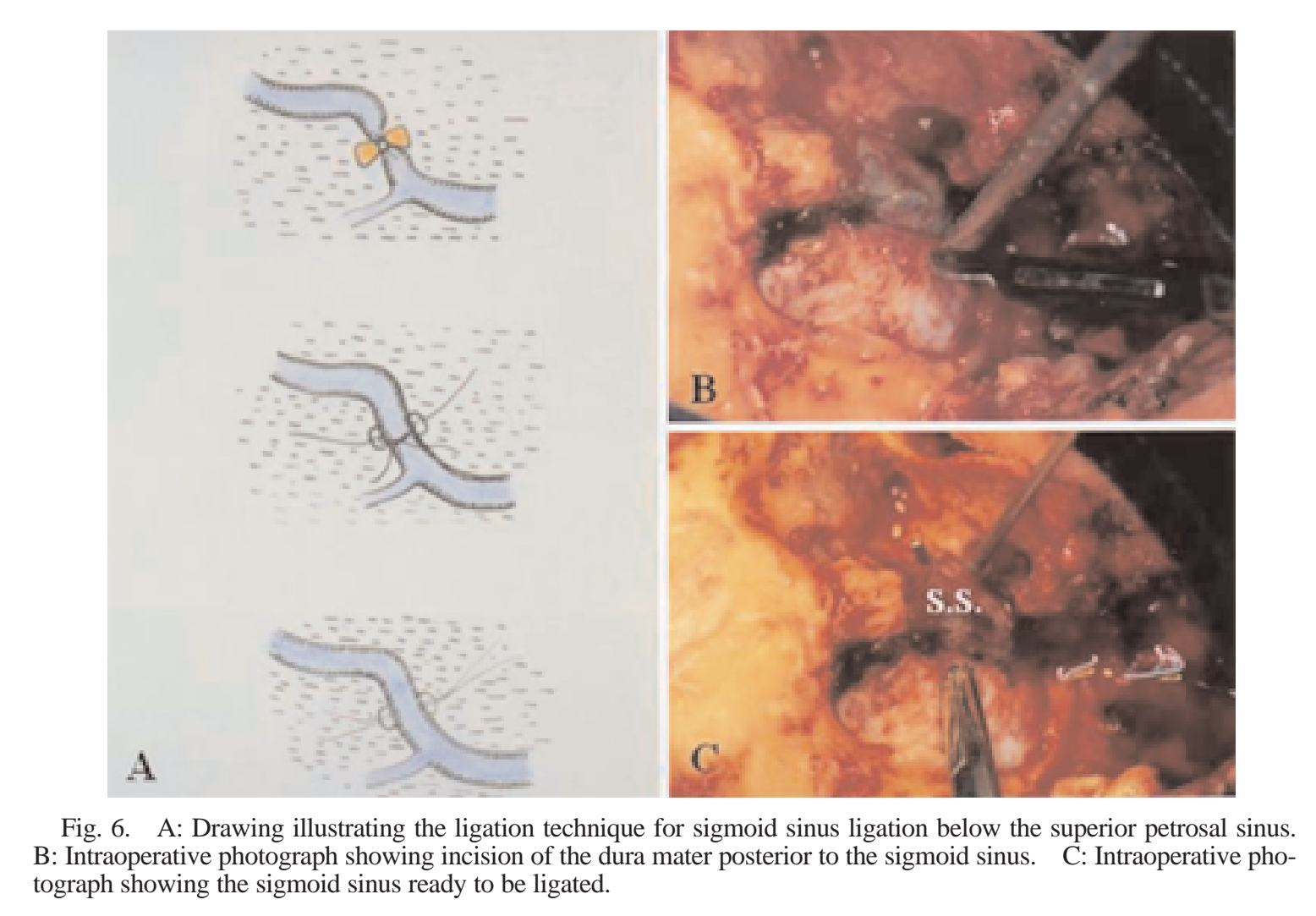

Surgical damage of lower cranial nerves was the most common and also the most dangerous complication. Ten patients $(9.8 \%)$ presented with this complication, and it was permanent in six. Facial and cochlear nerve paralysis occurred in eight cases each $(7.8 \%)$. Facial nerve palsy was temporary in three patients, and in the remaining five the facial nerve was reconstructed with nerve grafts (great auricular nerve) or 12th/seventh cranial nerve anastomosis. All these patients attained good functional recovery. Four patients (3.9\%) experienced CSF leakage. One underwent repeated surgery and three others were treated conservatively with lumbar drainage; in three of these patients meningitis developed. Two patients had cervical hematomas and one experienced hemiparesis.

Four patients $(3.9 \%)$ died after surgery. The main cause of death was surgical damage to lower cranial nerves complicated by aspiration pneumonia and septicemia (two patients). One patient died of a pulmonary embolism and one presented postoperatively with bleeding from the ligated IJV, which caused a large cervical hematoma, tracheal deviation, and cerebral hypoxia. This patient was intubated and the hematoma was removed, but the patient died of complications secondary to brain hypoxemia.

\section{ILLUSTRATIVE CASES}

\section{Patient With Paraganglioma}

This 35-year-old woman had suffered from left-sided pulsatile tinnitus for 1 year before admission to our clinic. The symptoms increased in intensity and she experienced left-sided hearing loss and swallowing difficulties. Ad- mission CT and MR imaging studies revealed a large tumor in the jugular foramen, ear, and cervical region with a small extension into the posterior fossa (Fig. 9). The neurological examination at admission showed left-sided deafness and hypesthesia of the left nasopharynx. The tumor was radically resected and no additional postoperative deficit occurred (Fig. 9).

\section{Patient With Schwannoma}

This 52-year-old man was admitted to our clinic with a history of surgery for jugular foramen schwannoma 5 years before. He reported swallowing difficulty and hoarseness. His neurological examination showed diminished hearing and paralysis of the ninth and 10th cranial nerves on the left side. Admission MR images revealed a large recurrent cystic jugular foramen lesion with extensions into the posterior fossa and cervical region (Fig. 10). The tumor was totally removed with no additional neurological deficits.

\section{Patient With Meningioma}

This 42-year-old woman presented with a 12-month history of hoarseness and swallowing difficulties. Her neurological examination revealed paralysis of the ninth and 10th right cranial nerves. An admission CT scan revealed a large, enhancing tumor in the right jugular foramen that contained calcifications. This tumor extended into the posterior fossa, internal auditory canal, and spinal canal (Fig. 11). The lesion was removed using a multidisciplinary approach and the postoperative course was uneventful. 


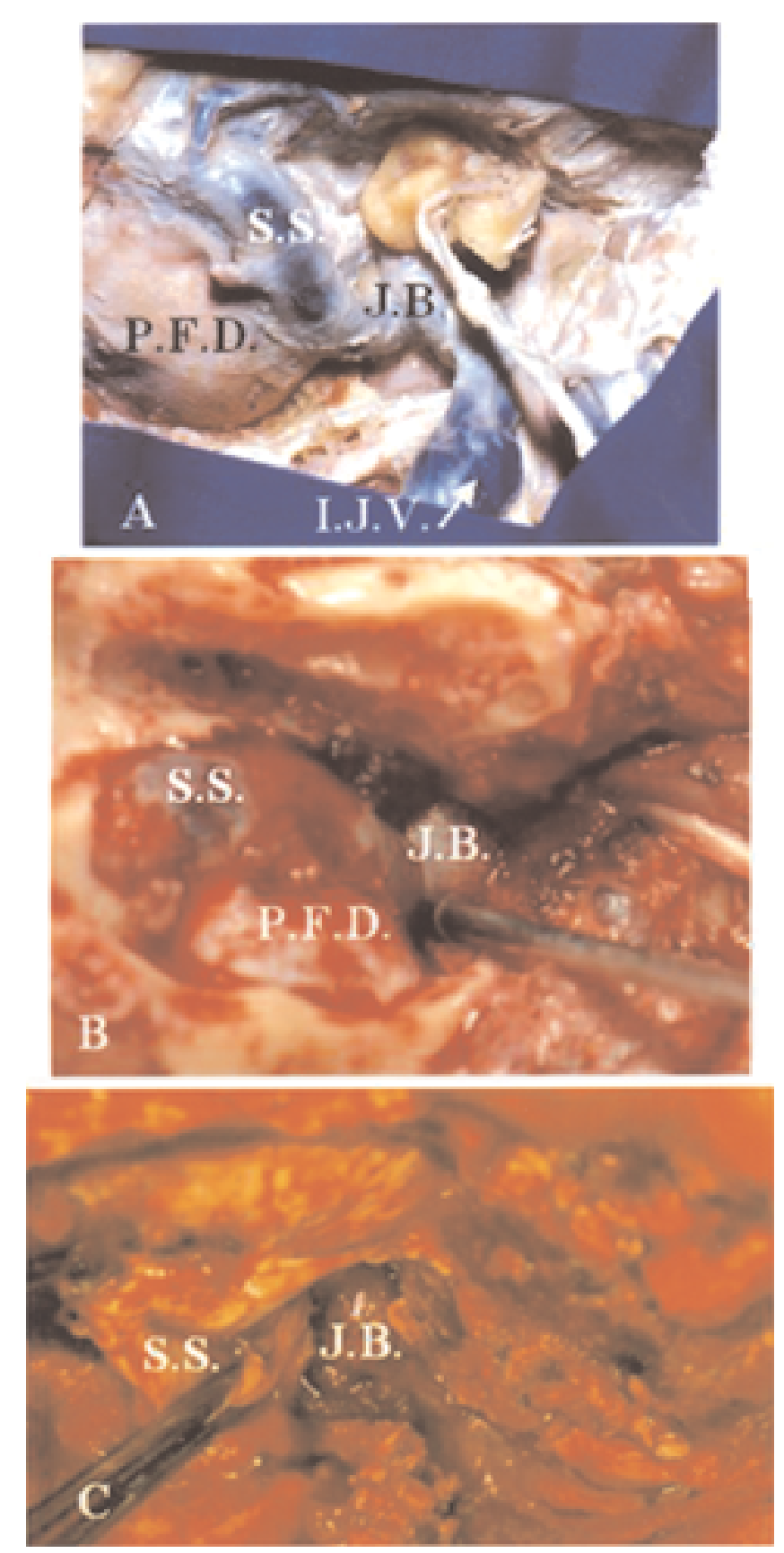

Fig. 7. A: Photograph of an anatomical specimen showing the posterior fossa dura (P.F.D.), jugular bulb (J.B.), sigmoid sinus, and the IJV. B: Intraoperative photograph demonstrating wide exposure of the jugular bulb, posterior fossa dura, and sigmoid sinus. C: Intraoperative photograph showing tumor removal from the sigmoid sinus and jugular bulb.

\section{DISCUSSION}

\section{Paragangliomas of the Jugular Foramen}

In 1941, Guild ${ }^{18}$ described carotid body-like structures in the temporal bone and named them "glomus jugulare bodies." In 1945, Rosenwasser ${ }^{40,41}$ published the first description of a middle ear paraganglioma and associated these tumors with the glomus jugulare bodies. In 1962,

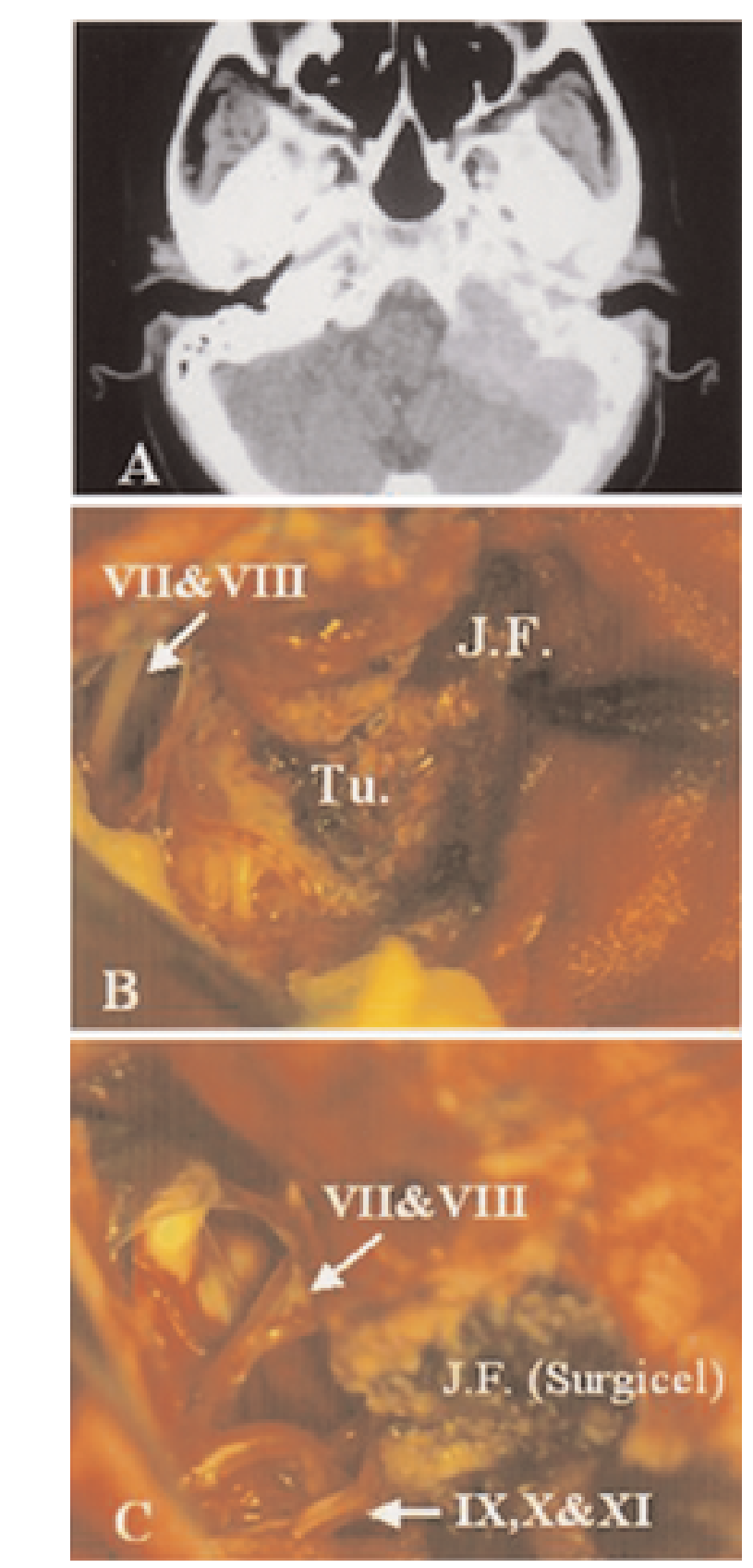

Fig. 8. A: Admission CT scan demonstrating a large jugular foramen paraganglioma. B: Intraoperative photograph showing surgical exposure of a large jugular foramen paraganglioma; the caudal cranial nerves are embedded in the tumor. C: Photograph obtained after removal of the lesion. The jugular foramen is occluded with Surgicel.

Alford and Guilford ${ }^{1}$ classified these lesions as glomus tympanicum and glomus jugulare tumors. Multiple terms have been used to describe paragangliomas-glomus tumor, chemodectoma, glomerocytoma, nonchromaffin tumor, tympanic body tumor, and receptoma are the most common. ${ }^{19}$

Chemoreceptor cells are nonchromaffin-staining paraganglion cells developed from the neural crest region during embryogenesis. ${ }^{46,51}$ These structures are found in the 


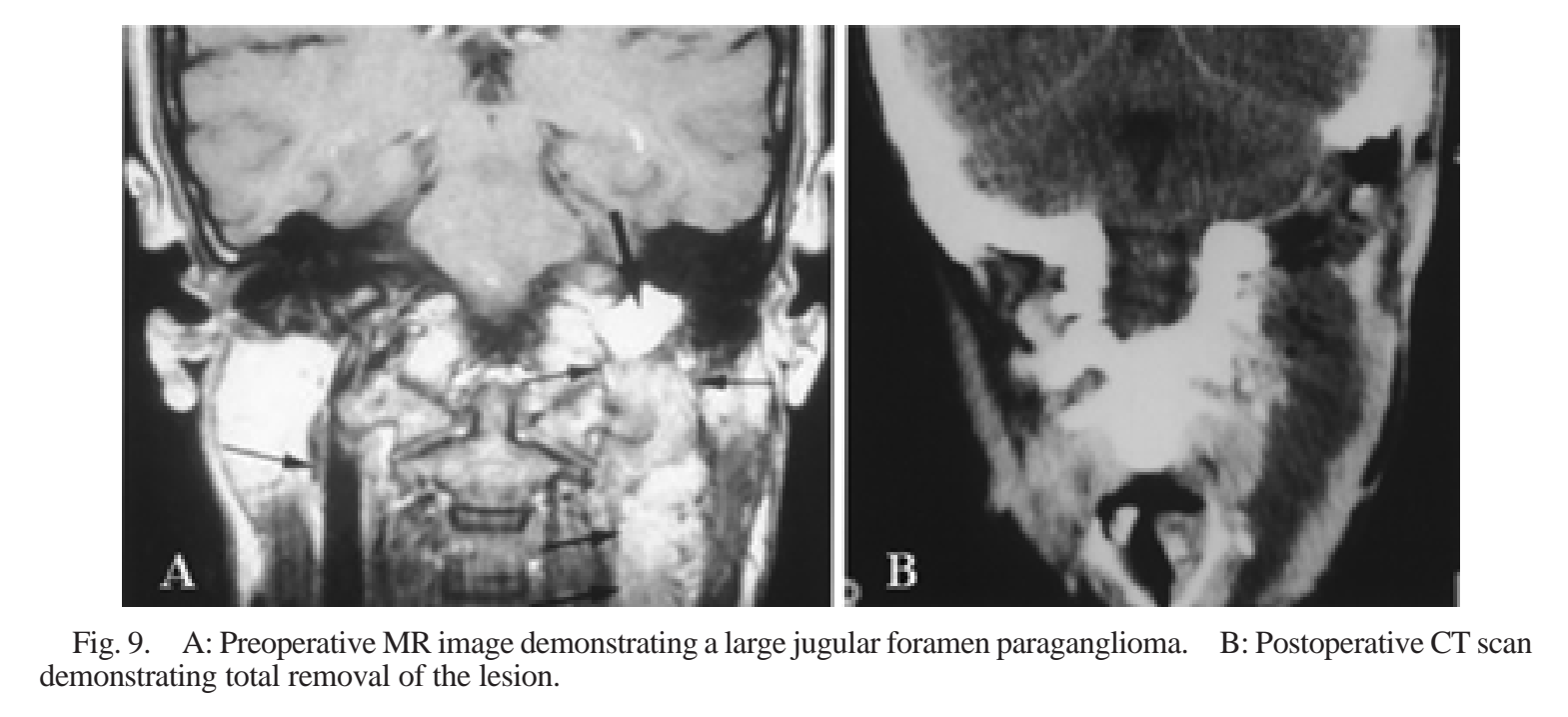

adventitia of the jugular bulb beneath the floor of the middle ear, in the bone walls of the tympanic canals related to the tympanic branches of the glossopharyngeal and vagus nerves, in the bone of promontory, close to the mucosal lining of the middle ear, in the ciliary ganglion, the ganglion nodosum of the vagus nerve, and in the walls of large arteries. ${ }^{10,31}$ The arterial blood supply to these bodies comes from the ascending pharyngeal artery through its inferior tympanic branch. The histological features of these bodies are the same in the glomus caroticum and glomus aorticum; histologically, paragangliomas are similar in all locations.

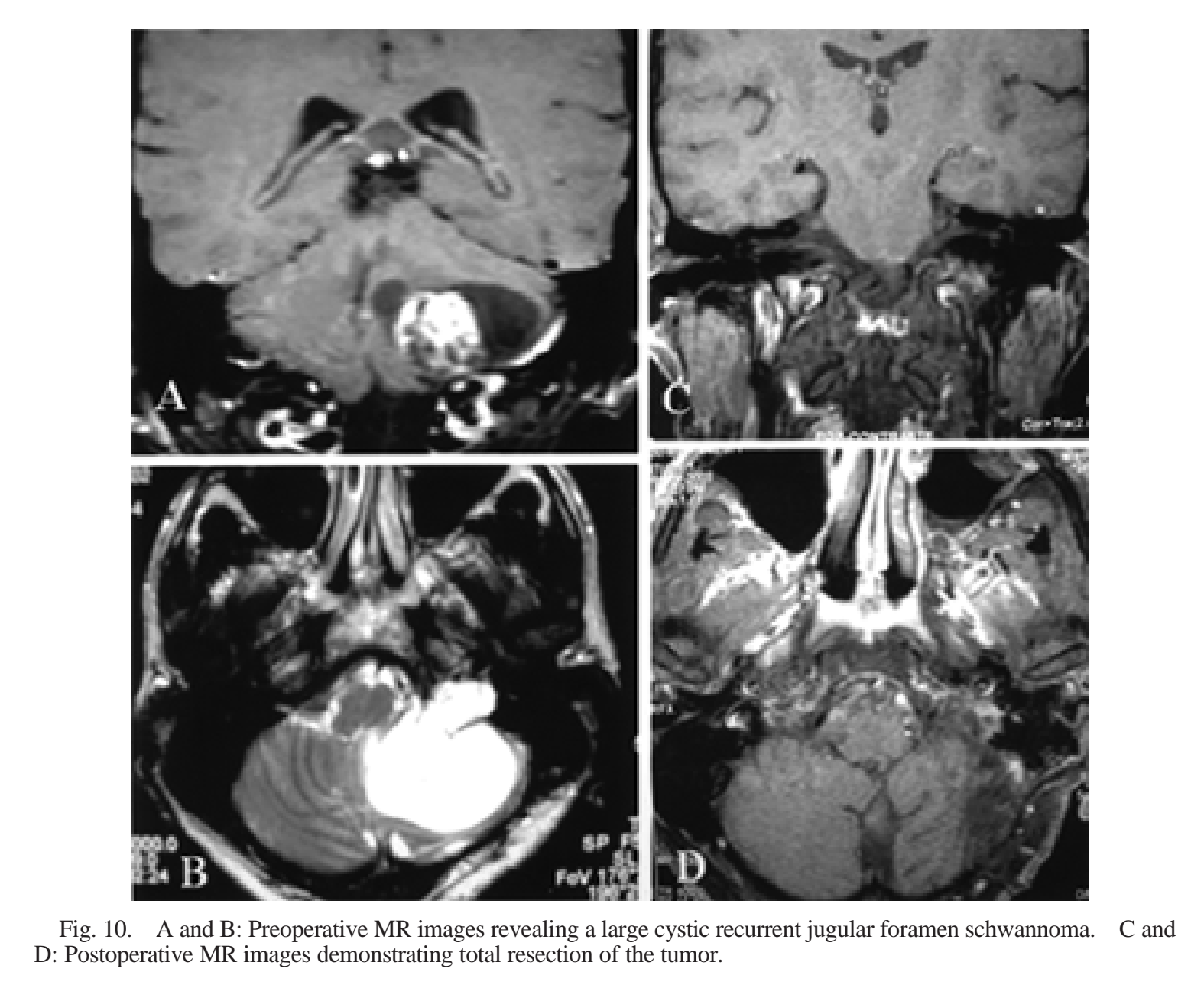



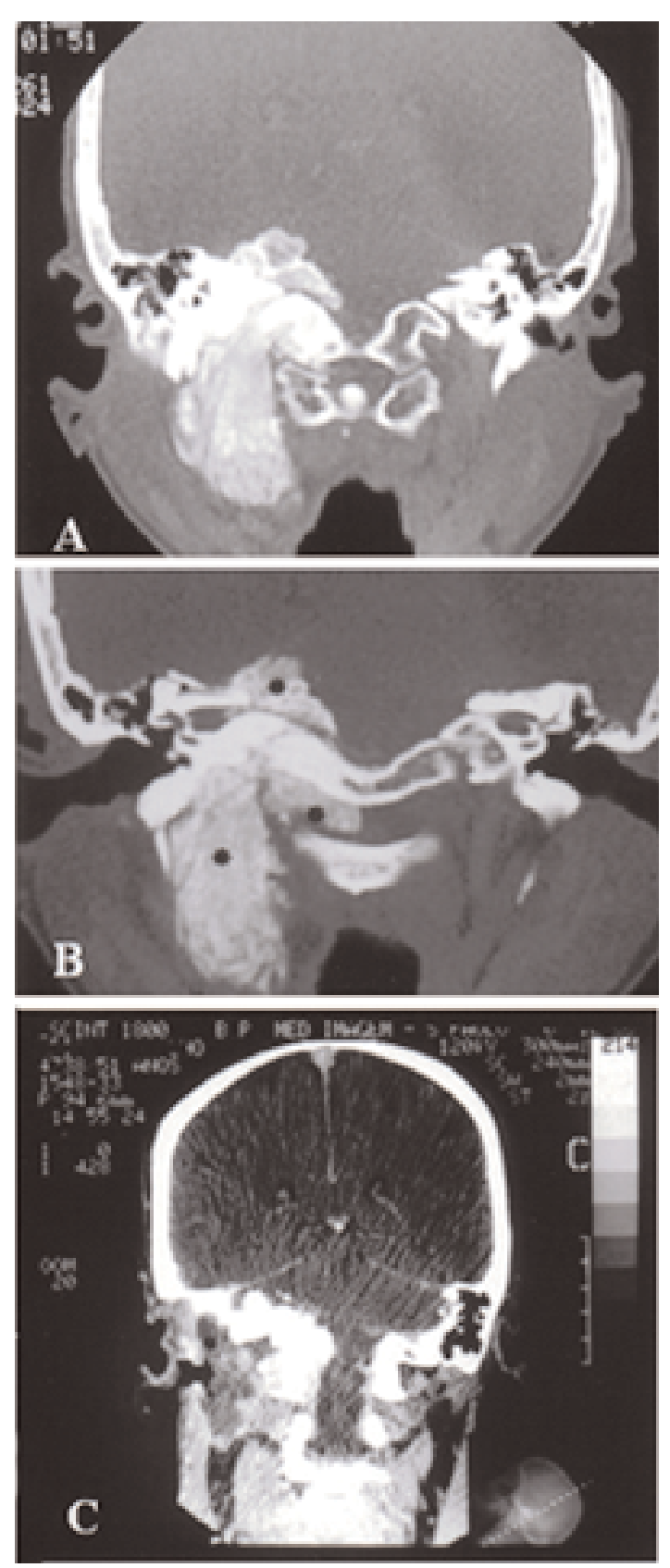

Fig. 11. A and B: Preoperative CT scan demonstrating a large jugular foramen meningioma with extension into the posterior fossa, internal auditory canal, and spinal canal. C: Postoperative CT scan obtained after resection of the tumor.

Familial cases present with a much higher incidence of multicentricity; 48 in these cases the paternal allele is the determining factor. ${ }^{49}$ There is a $50 \%$ incidence of male carrier offspring and $0 \%$ female carrier offspring with paraganglioma. This genetic abnormality is created by mutations located on chromosome 11 at $11 \mathrm{q} 13$ and q2. ${ }^{7}$ The most frequent initial symptoms are pulsatile tinnitus, hearing loss, and cranial nerve involvement. Diagnosis is usually made by ear, nose, and throat surgeons, and neurosurgeons usually see patients with large intracranial extensions. Hypertension, headache, arrhythmias, nausea, and palpitations occur when the tumor secretes norepinephrine. Perioperative treatment with $\beta$-blockers is necessary for these patients.

\section{Other Jugular Foramen Tumors}

Schwannomas of lower cranial nerves, meningiomas, chondrosarcomas, and high-grade malignant tumors are other lesions originating in the jugular foramen. Hearing loss and paralysis of lower cranial nerves are often the initial symptoms of these tumors. Most jugular foramen schwannomas are benign, noninfiltrative lesions. ${ }^{11}$ Meningiomas arise from arachnoid cells in the jugular bulb and infiltrate surrounding bone and nerve tissue. ${ }^{32}$ In this series all patients with meningiomas presented with lower cranial nerve deficits and those with malignant lesions had pain in the ear and mastoid region. Clinical signs and symptoms may not, however, accurately indicate the extension of the lesion.

\section{Surgical Treatment}

Surgical exposure of this region requires understanding of the complex jugular foramen anatomy. Anatomical studies have been conducted but the anatomy of this structure remains poorly understood ${ }^{6,24,29}$ Classically, the jugular foramen is described as consisting of two portions: the nervous part, containing the hypoglossal nerve, the inferior petrosal sinus, and the meningeal branches of the ascending pharyngeal artery; and the venous part, containing the sigmoid sinus and the vagus and accessory nerves. Anatomical studies have shown that the arrangement of the neurovascular structures within the jugular foramen does not conform to the compartmentalization into an anteromedial pars nervosa and a posterolateral pars venosa. ${ }^{6}$

Katsuta, et al., ${ }^{24}$ divided the jugular foramen into three portions: two venous and one nervous (intrajugular). The nervous portion is localized between the two venous ones and it contains the ninth, 10th, and 11th cranial nerves. The course of the cranial nerves within the jugular foramen presents many anatomical variations. Sen, et al. ${ }^{43}$ described the cranial nerves running anteromedially to the jugular bulb and maintaining a multifascicular histoarchitecture (particularly the 10th cranial nerve). The vagus nerve is formed by multiple fascicles and the glossopharyngeal and accessory nerves by one and two fascicles, respectively. The lower cranial nerves (ninth, 10th, and 11 th) run through a septum of connective tissue in continuity with the dura mater and the pericranium. The tympanic branch of glossopharyngeal nerve (Jacobson nerve) and the auricular branch of the vagus nerve (Arnold nerve) cross the jugular foramen.

Preoperative evaluation includes complete otological and neurological examination, CT scanning, MR imaging, MR angiography, and digital subtraction angiography in cases of highly vascularized lesions. Balloon test occlusion is performed if the ICA is involved or encased. A high-flow shunt (saphenous vein or radial artery graft) is used to reconstruct this vessel. Preoperative embolization is performed in cases of highly vascularized tumors, and is useful to reduce bleeding and surgical time..$^{33}$

Resection of these lesions is difficult for the following reasons: jugular foramen tumors are deeply located; some 
are highly vascularized and may involve or infiltrate cranial nerves, vessels, brainstem, and the bone structures at the cranial base, producing a large surgical defect; and some may present with major posterior fossa extension. Despite these difficulties, radical removal of a benign jugular foramen tumor may be curative and remains the treatment of choice. ${ }^{2,3,15,17,21,37,39,42}$ The size of the lesion is directly related to the complication rate. ${ }^{8}$ Total resection of benign paragangliomas with large intracranial extension may be impossible or associated with unacceptable complications. . $^{3,10,15}$ Paragangliomas may invade cranial nerves that still function, and total resection is only possible with sacrifice of these nerves. ${ }^{43}$

The multidisciplinary approach gives the best chance of radical removal with preservation of cranial nerves, vessels, and the brainstem. To avoid postoperative complications, an adequate surgical exposure and reconstruction of the cranial base are required. Surgical morbidity and mortality are usually associated with damage to the lower cranial nerves. ${ }^{8}$ Identification and dissection in the neck and at the brainstem is helpful in the preservation of these nerves. When they are infiltrated yet still functioning, our strategy is to leave a small piece of tumor around them and if necessary (if there is proven postoperative residual tumor growth) administer radiotherapy.

Two patients in this series died of aspiration pneumonia. Tracheotomy should be performed as early as possible if clinical signs of persistent aspiration are present. Cases of CSF leakage are common and may be a serious complication after resection of jugular foramen lesions. ${ }^{3}$, $22,25,50$ The cranial base is usually reconstructed with abdominal fat graft, rotation of local and distant pedicled muscle flaps, and free muscle flaps vascularized with microsurgical vessel anastomosis., $4,15,23,34,36$

Abdominal fat grafts are commonly used in skull base surgery. The procedure has some disadvantages: an additional skin incision is required, the fat tissue is not vascularized, a foreign body reaction may occur, and compression of the brainstem ${ }^{13}$ and cases of lipoid meningitis are described in the literature. ${ }^{20,38}$ Regional muscle flap rotation increases surgical time, necessitates additional incisions, and may produce poor cosmetic results. ${ }^{5,16,35}$ Free muscle flaps vascularized with microsurgical techniques have the following disadvantages: increased surgical time and risk of infection, an additional surgical team is required, and there is a higher morbidity rate in elderly patients. ${ }^{5,6,12}$

The reconstruction technique developed in our clinic has been used since 1987 (unpublished data) and presents several advantages when compared with the other procedures. It is easy to perform, vascularized flaps are used, it can be used for small and large defects, it causes no additional deficits, the cosmetic results are very good, it reduces the incidence of CSF leakage, and postoperative lumbar drainage is rarely needed. Postoperative lumbar drainage is frequently used in the surgery of skull base lesions, ${ }^{15,44}$ and this procedure may lead to serious morbidity. ${ }^{45}$

\section{Postoperative Treatment}

Some authors recommend radiotherapy as the initial treatment for paragangliomas, because resection of these tumors is associated with damage to the lower cranial nerves. ${ }^{30,47}$ Series in which more than $80 \%$ tumor control is reported after radiotherapy are presented. ${ }^{14,26,27}$ The effect of radiation appears to be on the blood vessels and fibrous elements of the lesion, $, 97,47$ and the beneficial effects of radiotherapy for these tumors remain unclear. ${ }^{17}$ Radiation-induced neoplasm has been described after radiotherapy for benign glomus jugulare tumors. ${ }^{28}$ In our series postoperative radiotherapy was indicated for elderly patients who are poor surgical candidates, for malignant or aggressive tumors, and for tumor remnants in the cavernous sinus that present with growth on postoperative MR images. Radiosurgery with gamma knife or linear accelerator modalities may be more beneficial in reducing the side effects of irradiation. Chemotherapy is indicated for some malignant lesions.

\section{CONCLUSIONS}

Paraganglioma was the most common tumor in this series. Pulsatile tinnitus and hearing loss were the most frequent initial symptoms in these patients. Familial cases presented with a higher rate of multiple tumors. Total resection of schwannomas, benign meningiomas, noninfiltrative paragangliomas, and other benign jugular foramen tumors was possible with low mortality rates, and may be curative. Radical resection of malignant and invasive tumors with preservation of cranial nerves was not possible. Postoperative morbidity and mortality were related to the size and histological features of the lesions. Radical resection of large paragangliomas of the jugular foramen with intracranial extension is difficult, and a multidisciplinary skull base approach offers the best chance of total removal with preservation of lower cranial nerves. Preoperative embolization was very useful in most cases of paragangliomas and well-vascularized tumors.

Aspiration and swallowing abnormalities caused by surgical damage to lower cranial nerves was the most dangerous complication related to surgery. Delayed extubation, tracheotomy in selected cases, and early rehabilitation are very important in the postoperative management of these tumors. Reconstruction of the skull base with vascularized myofascial flaps reduced postoperative CSF leaks and produced very good cosmetic results.

\section{References}

1. Alford BR, Guilford FR: A comprehensive study of tumors of the glomus jugulare. Laryngoscope 72:765-805, 1962

2. Al-Mefty O, Fox JL, Rifai A, et al: A combined infratemporal and posterior fossa approach for the removal of giant glomus tumors and chondrosarcomas. Surg Neurol 28:423-31, 1987

3. Al-Mefty O, Teixeira A: Complex tumors of the glomus jugulare: criteria, treatment, and outcome. J Neurosurg 97: 1356-1366, 2002

4. Anand V: Reconstruction in cranial base surgery, in Al-Mefty O (ed): Surgery of the Cranial Base. Boston: Kluwer Academic Publishers, 1989, pp 297-314

5. Ariyan $\mathrm{S}$ : Pectoralis major muscle and musculocutaneous flaps, in Strauch B, Vasconez LO, Hall-Findlay EJ (eds): Grabb's Encyclopedia of Flaps. Boston: Little, Brown, 1990, pp 512-522

6. Ayeni SA, Ohata K, Tanaka K, et al: The microsurgical anatomy of the jugular foramen. J Neurosurg 83:903-909, 1995 
7. Bikhazi PH, Roeder E, Attaie A, et al: Familial paragangliomas: the emerging impact of molecular genetics on evaluation and management. Am J Otol 20:639-643, 1999

8. Bowdino B, Farrell P, Moore G, et al: Long-term surgical results for glomus temporale tumors. Neurosurg Q 14:19-26, 2004

9. Brackmann DE, House WF, Terry R, et al: Glomus jugulare tumors: effect of irradiation. Trans Am Acad Ophthalmol Otolaryngol 76:1423-1431, 1972

10. Brown JS: Glomus jugulare tumors revisted: a ten-year statistical follow-up of 231 cases. Laryngoscope 95:284-288, 1985

11. Carvalho GA, Tatagiba M, Samii M: Cystic schwannomas of the jugular foramen: clinical and surgical remarks. Neurosurgery 46:560-566, 2000

12. Chang DW, Langstein HN, Gupta A, et al: Reconstructive management of cranial base defects after tumor ablation. Plast Reconstr Surg 107:1346-1357, 2001

13. Chen TC, Maceri DR, Levy ML, et al: Brain stem compression secondary to adipose graft prolapse after translabyrinthine craniotomy: case report. Neurosurgery 35:521-524, 1994

14. Cummings BJ, Beale FA, Garrett PG, et al: The treatment of glomus tumors in the temporal bone by megavoltage radiation. Cancer 53:2635-2640, 1984

15. Fisch U: Infratemporal fossa approach for glomus tumors of the temporal bone. Ann Otol Rhinol Laryngol 91:474-479, 1982

16. Gal TJ, Kerschner JE, Futran ND, et al: Reconstruction after temporal bone resection. Laryngoscope 108:476-481, 1998

17. Green JD Jr, Brackmannn DE, Nguyen CD, et al: Surgical management of previously untreated glomus jugulare tumors. Laryngoscope 104:917-921, 1994

18. Guild SR: A hitherto unrecognized structure, the glomus jugularis, in man. Anat Rec 79 (Suppl 1):28, 1941 (Abstract)

19. Gulya AJ: The glomus tumor and its biology. Laryngoscope 103 (Suppl 60):7-15, 1993

20. Hwang PH, Jackler RK: Lipoid meningitis due to aseptic necrosis of a free fat graft placed during neurotologic surgery. Laryngoscope 106:1482-1486, 1996

21. Jackson CG, Glasscock ME III, Nissen AJ, et al: Glomus tumor surgery: the approach, results, and problems. Otolaryngol Clin North Am 15:897-916, 1982

22. Jackson CG, Woods CI, Chironis PN: Glomus jugulare tumors, in Sekhar LN, Janecka IP (eds): Surgery of Cranial Base Tumors. New York: Raven Press, 1993, pp 747-768

23. Jones NF, Schramnn VL, Sekhar LN: Reconstruction of the cranial base following tumour resection. Br J Plast Surg 40: $155-162,1987$

24. Katsuta T, Rhoton AL Jr, Matsushima T: The jugular foramen: microsurgical anatomy and operative approaches. Neurosurgery 41:149-202, 1997

25. Kempe LG: Glomus jugulare tumor, in Youmans JR (ed): Neurological Surgery, ed 2. Philadelphia: WB Saunders, 1982, pp 3285-3298

26. Kim JA, Elkon D, Lim ML, et al: Optimum dose of radiotherapy for chemodectomas of the middle ear. Int J Radiol Oncol Biol Phys 6:815-819, 1980

27. Konefal JB, Pilepich MV, Spector GJ, et al: Radiation therapy in the treatment of chemodectomas. Laryngoscope 97: 1331-1335, 1987

28. Lalwani AK, Jackler RK, Gutin PH: Lethal fibrosarcoma complicating radiation therapy for benign glomus jugulare tumor. Am J Otol 14:398-402, 1993

29. Lang J, Samii M, Draf W: Surgery of the Skull Base: An Interdisciplinary Approach. Berlin: Springer-Verlag, 1989, pp 59-64

30. Larner JM, Hahn SS, Spaulding CA, et al: Glomus jugulare tumors. Long-term control by radiation therapy. Cancer 69: 1813-1817, 1992
31. Lattes R, Waltner JG: Nonchromaffin paraganglioma of the midde ear. Cancer 2:447-468, 1949

32. Molony TB, Brackmann DE, Lo WW: Meningiomas of the jugular foramen. Otolaryngol Head Neck Surg 106:128-136, 1992

33. Murphy TP, Brackmann DE: Effects of preoperative embolization on glomus jugulare tumors. Laryngoscope 99:1244-1247, 1989

34. Mustoe TA, Corral CJ: Soft tissue reconstructive choices for craniofacial reconstruction. Clin Plast Surg 22:543-554, 1995

35. Neligan PC, Mulholland S, Irish J, et al: Flap selection in cranial base reconstruction. Plast Reconstr Surg 98:1159-1168, 1996

36. Netterville JL, Civantos EJ: Defect reconstruction following neurotologic skull base surgery. Laryngoscope 103 (Suppl 60):55-63, 1993

37. Ramina R, Maniglia JJ, Pedrozo AA, et al: [Surgical treatment of jugular foramen tumors.] Zentralbl Neurochir 57 (Suppl 1):47, 1996 (Ger) (Abstract) (Reference unverified)

38. Ricaurte JC, Murali R, Mandell W: Uncomplicated postoperative lipoid meningitis secondary to autologous fat graft necrosis. Clin Infect Dis 30:613-615, 2000

39. Robertson JT, Clark WC, Robertson JH, et al: Glomus jugulare tumors, in Youmans JR (ed): Neurological Surgery, ed 3. Philadelphia: WB Saunders, 1990, pp 3654-3666

40. Rosenwasser H: Carotid body like tumor involving the middle ear and mastoid bone. Arch Otolaryng 41:64-67, 1945

41. Rosenwasser H: Glomus jugularis tumor of the middle ear; carotid body tumor, tympanic body tumor, nonchromaffin paraganglioma. Laryngoscope 62:623-633, 1952

42. Samii M, Tatagiba M: Tumors of the jugular foramen. Neurosurg Q 6: 176-193, 1996

43. Sen C, Hague K, Kacchara R, et al: Jugular foramen: microscopic anatomic features and implications for neural preservation with reference to glomus tumors involving the temporal bone. Neurosurgery 48:838-848, 2001

44. Shapiro SA, Scully T: Closed continuous drainage of cerebrospinal fluid via a lumbar subarachnoid catheter for treatment or prevention of cranial/spinal cerebrospinal fluid fistula. Neurosurgery 30:241-245, 1992

45. Snow RB, Kuhel W, Martin SB: Prolonged lumbar spinal drainage after the resection of tumors of the skull base: a cautionary note. Neurosurgery 28:880-883, 1991

46. Spector GJ, Ciralsky R, Maisel RH, et al: IV. Multiple glomus tumors in the head and neck. Laryngoscope 85:1066-1075, 1975

47. Springate SC, Weichselbaum RR: Radiation or surgery for chemodectoma of the temporal bone: a review of local control and complications. Head Neck 12:303-307, 1990

48. van Baars F, van den Broek P, Cremers C, et al: Familial nonchromaffinic paragangliomas (glomus tumors): clinical aspects. Laryngoscope 91:988-996, 1981

49. van der Mey AG, Maaswinkel-Mooy PD, Cornelisse CJ, et al: Genomic imprinting in hereditary glomus tumors: evidence of a new genetic theory. Lancet 2:1291-1294, 1989

50. Watkins LD, Mendoza N, Cheesman AD, et al: Glomus jugulare tumours: a review of 61 cases. Acta Neurochir 130:66-70, 1994

51. Zak FG: An expanded concept of tumors of glomic tissue. N Y State J Med 54:1153-1165, 1954

Manuscript received June 21, 2004.

Accepted in final form July 15, 2004.

Address reprint requests to: Ricardo Ramina, M.D., R. Goncalves Dias 713, 80.340-240 Curitiba, Brazil. email: ramina@ inc-neuro.com.br. 